\title{
On stress reconstruction in composite domains from discrete data on principal directions
}

\author{
A. N. Galybin \\ Wessex Institute of Technology, Southampton, UK
}

\begin{abstract}
The problem considered in this paper deals with reconstruction of plane stress tensors in domains with multiple interfaces representing the boundaries of subdomains. No boundary stresses or displacements are imposed; instead the discrete data on stress orientations are used for problem setting. Continuity of the stress vector across the interfaces is also accepted. Solutions in every subdomain are sought by assuming linear approximations for the complex potentials with unknown coefficients. These are found by solving a minimisation problem. An application to the geophysical problem of stress identification in tectonic plates is considered to illustrate effectiveness of the proposed numerical approach.
\end{abstract}

Keywords: stress orientations, $2 D$ stress tensor, complex potentials, plate tectonics.

\section{Introduction}

The problem of stress reconstruction from discrete data on stress orientation has been addressed in [1, 2] for a single domain and in [3-4] for two subdomains. In all these cases the domains have been associated with either tectonic plates or with certain regions of the earth's crust and real data on stress orientations supplied by the World Stress Map Project [5] have been used. The present study is aimed at the further development of the previously reported method for the case when the entire domain consists of several plane elastic subdomains whose deformation properties may be, in general, different.

This study is also aimed at the development of algorithms capable of dealing with the data widely used in geodynamics, namely, experimentally obtained stress orientations around the globe and geographical coordinates of the tectonic plate margins [6]. 
The paper is structured as follows. Typical data accepted in this study are described in section 2 where also the outcomes of the developed algorithms are presented. Section 3 presents mathematical formulation of the problem and its numerical implementation is described in section 4. Results of computations for a particular plane domain are discussed in section 5 .

\section{Data}

Data on stress orientations have been extracted from the WSM database which current 2008 release contains 21,750 stress data records, [5]. Only data of A-B quality [7] are used further on, this assumes stress orientations are recorded with the following errors: $\pm 10^{\circ}-15^{\circ}$ (A-quality) and within $\pm 15^{\circ}-20^{\circ}$ (B-quality).

Data on plate boundaries [6] contains 52 entries. Each plate is presented by an ordered set of boundary points (in geographical coordinates) that constitutes a closed curve traversed in the counterclockwise direction. The last point coincides with the first point.

A fragment of the earth's crust with the data is shown in Fig 1. The domain consists of 16 subdomains (presenting tectonic plates); short segments show 53 orientations of major compressive principal stresses. It is evident that the data are highly irregular and not all subdomains contain data points.

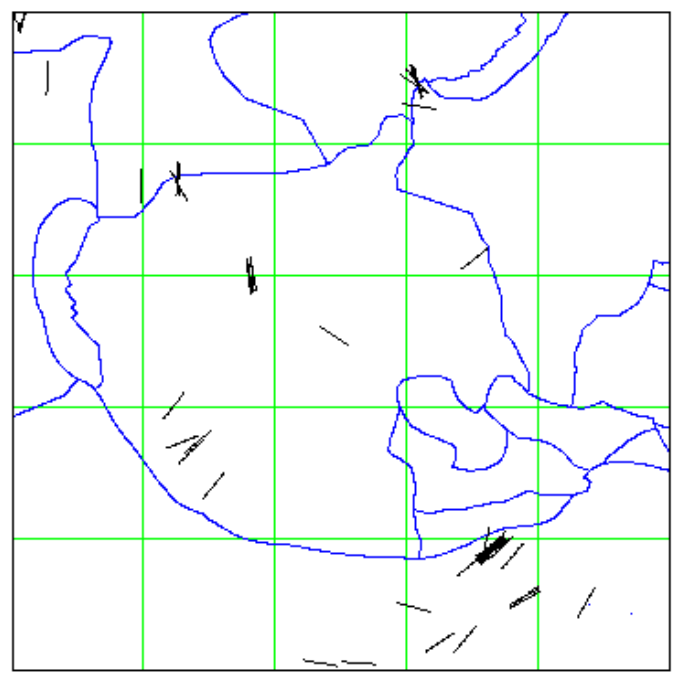

Figure 1: Domain consisting of 16 subdomains, 35 interfaces, 53 data on stress orientations.

Several special procedures have been designed to deal with the boundary data with the aim of identification of the interfaces between the subdomains and placing equidistant collocation points on the interfaces. The number of interfaces depends on a particular subdomain pattern and cannot be calculated in a simple 
way as it can be done for regular grids. For instance, 35 interfaces have been identified for the example presented in Fig 1. They represent common boundaries between adjacent subdomains and therefore there is one-to one correspondence between an interface number and a pair of subdomain numbers.

Linear interpolation has been applied through the discrete points representing an interface; therefore a set of nodes has been introduced for each interface that is further used for forming the entire set of collocation points. The number of nodes in every set is different, it is defined by the length, $L_{j}$, of the $j$-th interface $\left(j=1 \ldots N_{\text {int }}\right)$ and the step, $\Delta$, specified as a global input parameter; therefore the number of nodes on the $j$-th interface is defined as $\operatorname{Nnode}_{j}=$ floor $\left(L_{j} / \Delta\right)$.

After initial treatment of data the following output is obtained:

a. The table of correspondence between interfaces and subdomains; it is a two row array with $N_{\text {int }}$ columns, its first column shows the interface number and its second column contains the numbers of two adjacent subdomains whose boundaries form the interface shown in the first column;

b. complex coordinates of the nodes on all interfaces $\zeta_{k}, k=1 \ldots \sum_{j=1}^{N \text { int }} N n o d e_{j}$

c. principal directions, angles $\theta_{m}$, at points $z_{m}, m=1 \ldots N_{\text {data }}$; and

d. complex coordinates of the data points $z_{m}, m=1 \ldots N_{\text {data }}$ sorted with respect to subdomains.

A special procedure for the identification of the subdomain to which a particular datum belongs to has been designed for data sorting. This procedure is further used for mapping of the calculated stress field.

\section{Formulation of the problem}

A plane elastic domain $\Omega$ composed by the union of plane subdomains $\Omega_{i}$ $(i=1 \ldots N)$ having, in general, different elastic moduli is considered (elastic moduli are not specified because no conditions regarding discontinuity of displacements across the interfaces are assumed in formulation). Principal directions are given at some points, belonging to some subdomains (not necessarily all subdomains are populated with data); the discrete set of given principal directions as assumed to be finite and contain $N_{\text {data }}$ points. It is accepted that the stress vector is continuous across the interfaces $\Gamma_{j}$ between all adjacent subdomains $\left(j=1 \ldots N_{\text {int }}\right)$. Given these conditions it is required to determine all possible stress states satisfying the given data on principal directions.

It should be noted that the problem formulated above is not a classical boundary value problem of plane elasticity. In contrast to classical formulations, the number of boundary conditions (two for each interface, presenting continuity of the stress vector) is insufficient, and therefore the solution is not unique and may contain arbitrary functions. In order to overcome such high indeterminedness one should seek possible solutions in a certain class of functions. A simple set of basis functions, namely piecewise linear holomorphic functions, is used further in numerical implementation. However this does not make the problem to be fully well posed, there still may be a finite number of 
free parameters included in the solution. This is evident from the fact that no stress magnitudes are involved in the formulation, therefore at least two parameters remains undetermined in the total solution. They are (i) a multiplicative constant at the stress deviator; and (ii) an additive constant to the mean hydrostatic stress. These can be selected randomly and affect neither principal directions nor equilibrium equations. It has been shown [8] that up to 5 free parameters can be included in the total solution for a simply connected elastic isotropic domain when it is being built from the know stress trajectory pattern. For a composite domain considered here no theoretical results regarding the number of free parameters are available. Therefore, the presence of free parameters will further be verified by assessing the rank of the matrix of the corresponding linear system of equations.

General solution for the stress tensor for a plane elastic domain subjected to boundary forces only has the form [9]

$$
\begin{aligned}
& P(z, \bar{z}) \equiv \frac{\sigma_{11}+\sigma_{22}}{2}=\Phi(z)+\overline{\Phi(z)} \\
& D(z, \bar{z}) \equiv \frac{\sigma_{22}-\sigma_{11}}{2}+i \sigma_{12}=\bar{z} \Phi^{\prime}(z)+\Psi(z)
\end{aligned}
$$

Here $\Phi(z)$ and $\Psi(z)$ are complex potentials (holomorphic functions) to be determined from boundary conditions; harmonic function $P$ and complex-valued function $D$ represent mean hydrostatic stress and stress deviator respectively, they are functions of stress components $\sigma_{i j} ; z .=x_{1}+i x_{2}$ is a complex coordinate, over-bar stands for complex conjugation.

The complex conjugated stress vector $N(\zeta)-i T(\zeta)$ (where $N(\zeta)$ and $T(\zeta)$ are normal and shear components respectively) on an arbitrary interface $\Gamma_{j}$ is determined as follows [9]

$$
N(\zeta)-i T(\zeta)=P(\zeta)+\frac{d \zeta}{d \bar{\zeta}} D(\zeta), \quad \zeta \in \Gamma_{j}, \quad j=1 \ldots N_{\text {int }}
$$

where $P(\zeta)$ and $D(\zeta)$ are the boundary values of the stress function obtained by limiting transition of the field variable, $z$, to a boundary point, $\zeta$. These values can suffer jumps when the point crosses interfaces, however, the stress vector is assumed to be continuous across every interface, which leads to the following boundary condition in terms of complex potentials

$$
2 \operatorname{Re}\langle\Phi(\zeta)\rangle+\zeta \frac{d \zeta}{d \bar{\zeta}}\left\langle\Phi^{\prime}(\zeta)\right\rangle+\frac{d \zeta}{d \bar{\zeta}}\langle\Psi(\zeta)\rangle=0, \quad \zeta \in \Gamma_{j}, \quad j=1 \ldots N_{\text {int }}
$$

where $<\ldots>$ denoted the jump of the quantity across the $\mathrm{j}$-th interface.

The second set of conditions expresses the fact that the maximum shear stress, $\tau_{\max }=|D|$, is a real valued function, that allows one to write

$$
\operatorname{Im}\left[e^{2 i \theta_{m}}\left(\bar{z}_{m} \Phi^{\prime}\left(z_{m}\right)+\Psi\left(z_{m}\right)\right)\right]=0, \quad z_{m} \in \Omega, \quad m=1 \ldots N_{\text {data }}
$$

It is taken into account here that $\arg (D)=\pi-2 \theta$, where $\theta$ is principal direction (the angle between the major principal stress and the real axis) at an arbitrary point. 


\section{Numerical approach}

Complex potential are further sought as piecewise holomorphic linear functions

$$
\Psi(z)=\sum_{i=1}^{N} H_{i}(z), \quad \Phi(z)=\sum_{i=1}^{N} H_{N+i}(z)
$$

where $H_{i}(z)$ are linear in $\Omega_{i}$ and vanish outside

$$
H_{i}(z)=\left\{\begin{array}{cc}
c_{0}^{(i)}+c_{1}^{(i)} z & z \in \Omega_{i} \\
0 & z \notin \Omega_{i}
\end{array}, \quad H_{N+i}(z)=\left\{\begin{array}{ccc}
\frac{1}{2} a^{(i)}+c_{2}^{(i)} z & z \in \Omega_{i} \\
0 & z \notin \Omega_{i}
\end{array}, \quad i=1 \ldots N\right.\right.
$$

Here $c_{l}^{(i)}, l=0,1,2$ are complex constants while $a^{(i)}$ are real.

It follows from (1), (5),(6) that the stress functions assume the form

$$
\begin{aligned}
& D(z, \bar{z})=\sum_{i=1}^{N} D^{(i)}(z, \bar{z}), \quad D^{(i)}(z, \bar{z})=\left\{\begin{array}{cc}
c_{0}^{(i)}+c_{1}^{(i)} z+c_{2}^{(i)} \bar{z} & z \in \Omega_{i} \\
0 & z \notin \Omega_{i}
\end{array}\right. \\
& P(z, \bar{z})=\sum_{i=1}^{N} P^{(i)}(z, \bar{z}), \quad P^{(i)}(z, \bar{z})=\left\{\begin{array}{cc}
a^{(i)}+c_{2}^{(i)} z+\bar{c}_{2}^{(i)} \bar{z} & z \in \Omega_{i} \\
0 & z \notin \Omega_{i}
\end{array}\right.
\end{aligned}
$$

Although expressions (7) are simple they are general enough to address the presence of the singular (isotropic) points of two types known from photoelasticity [10]. The type of isotropic points is distinguished in accordance with the asymptotics near the roots of the stress deviator function [11].

There are seven unknown real coefficients for every subdomain, which are further considered as the coefficients in an ordered row as follows

$$
\mathbf{C}^{(i)}=\left(a^{(i)}, \operatorname{Re} c_{0}^{(i)}, \operatorname{Im} c_{0}^{(i)}, \operatorname{Re} c_{1}^{(i)}, \operatorname{Im} c_{1}^{(i)}, \operatorname{Re} c_{2}^{(i)}, \operatorname{Im} c_{2}^{(i)}\right), \quad i=1 \ldots N
$$
$\mathbf{C}^{(i)}$

These form the vector of unknowns $\mathbf{C}$ as a transpose of the augment of rows

$$
\mathbf{C}^{T}=\left(\mathbf{C}^{(1)}, \mathbf{C}^{(2)}, \ldots, \mathbf{C}^{(N)}\right)
$$

The first set of equations is obtained by satisfying the continuity condition (3) at collocation points, $\zeta_{k}$ as follows

$$
\mathbf{M}_{\mathbf{C o n}}=\left(\mathbf{U}^{(1)}, \mathbf{U}^{(1)}, \ldots \mathbf{U}^{\left(N_{\text {int }}\right)}, \mathbf{E}\right)^{T}, \quad \mathbf{U}^{(n)}=\left(\begin{array}{c}
0, \ldots, \operatorname{Re} \mathbf{V}^{(k)}, 0 \ldots, \operatorname{Re} \mathbf{V}^{(j)}, \ldots, 0 \\
0, \ldots, \operatorname{Im} \mathbf{V}^{(k)}, 0 \ldots, \operatorname{Im} \mathbf{V}^{(j)}, \ldots, 0
\end{array}\right)^{T}
$$

where $n$ is the number of the interface between the $k$-th and $j$-th subdomains, calculated in accordance with the correspondence table mentioned in section 2; and the vectors $\mathbf{V}^{(k)}$ and $\mathbf{E}$ are defined as follows

$$
\begin{aligned}
& \mathbf{V}^{(k)}=\left(1, \frac{d \zeta_{k}}{d \bar{\zeta}_{k}}, i \frac{d \zeta_{k}}{d \bar{\zeta}_{k}}, \zeta_{k} \frac{d \zeta_{k}}{d \bar{\zeta}_{k}}, i \zeta_{k} \frac{d \zeta_{k}}{d \bar{\zeta}_{k}}, 2 \operatorname{Re} \zeta_{k}+\frac{d \zeta_{k}}{d \bar{\zeta}_{k}},-2 \operatorname{Im} \zeta_{k}+i \bar{\zeta}_{k} \frac{d \zeta_{k}}{d \bar{\zeta}_{k}}\right) \\
& \mathbf{E}=(1,0,0,0,0,0,1,0, \ldots, 1,0,0,0,0,0,0)^{T}
\end{aligned}
$$


The vector $\mathbf{E}$ has been introduced to add an extra equation expressing the fact that the coefficients $a^{(i)}, i=1 \ldots N$ are not independent, which is a consequence of the field data type that does not include magnitudes of stresses in formulation.

The right-hand side corresponding the first set of equation has the form

$$
\mathbf{R}_{\text {Con }}=(0,0, \ldots, 0, \lambda)^{T}
$$

where the coefficient $\lambda$ can be chosen arbitrary, in particular, set to zero.

The second set of equations addresses the field data and employs conditions (4), which lead to the following matrix

$$
\mathbf{M}_{\text {data }}=\left(\mathbf{W}^{(1)}, \mathbf{W}^{(2)}, \ldots \mathbf{W}^{\left(N_{\text {data }}\right)}, \mathbf{J}\right)^{T}, \quad \mathbf{W}^{(m)}=\left(0, \ldots, \operatorname{Im} \mathbf{V}^{(m)}, \ldots, 0\right)^{T}
$$

where the vectors $\mathbf{V}^{(m)}$ and $\mathbf{J}$ are as follows

$$
\begin{aligned}
& \left.\mathbf{V}^{(m)}=\left(0,-e^{2 i \theta_{m}}, i e^{2 i \theta_{m}}, z_{m} e^{2 i \theta_{m}}, i z_{m} e^{2 i \theta_{m}}, \bar{z}_{m} e^{2 i \theta_{m}}, i \bar{z}_{m} e^{2 i \theta_{m}}\right)\right) \\
& \mathbf{J}=(0,1,1,1,1,1,1,0,1 \ldots, 0,1,1,1,1,1,1)^{T}
\end{aligned}
$$

The latter vector has been introduced into the system in order to make it inhomogeneous and acknowledge the fact that only the normalised maximum shear stress can be determined, i.e. one multiplicative arbitrary positive constant remains unknown.

The total system is obtained by the union of (10)-(14) as follows

$$
\mathbf{M C}=\mathbf{b}, \quad \mathbf{M}=\left(\begin{array}{l}
\mathbf{M}_{\text {Con }} \\
\mathbf{M}_{\text {data }}
\end{array}\right), \quad \mathbf{b}=\left(\begin{array}{l}
\mathbf{R}_{\text {Con }} \\
\mathbf{R}_{\text {data }}
\end{array}\right)
$$

The system is overdetermined it has $2 N_{\text {int }}+N_{\text {data }}+2$ equations and $7 N$ unknowns. Therefore its approximate solution is found by the least squares method (consistency is cheeked by calculating the rank of the matrix).

As soon as the coefficients $c_{k}$ are found the solutions for each subdomains are determined by the stress functions in (7).

\section{Model example}

This section presents an example of stress field reconstruction in a plane quadratic domain consisting of 9 subdomains as shown in Fig 2. The number of interfaces identified for this configuration is 18. The length of interfaces varies between 0.1 and 1.32 with the average value of 0.54 making the total length of all interfaces of 9.77. The number of collocation points introduced on the interfaces is 105 , they are shown in Fig. 2 by circles. The number of data on principal stresses is 116, the data has been taken from the WSMP [5] from the geographical domain between longitudes $-95^{\circ}$ to $-35^{\circ}$ and latitudes $-20^{\circ}$ to $30^{\circ}$; the only quality A-B has been selected. Two subdomains contain no data, and 4 subdomains have 1-2 data. The SLAE has 329 equations imposed on 63 unknowns.

The results of calculations of the stress field are presented in Figs. 3-5. Fig. 3 shown the map of normalised maximum shear stress, i.e. the function $F(z)$ in the expression $\tau_{\max }(\mathrm{z})=\mu F(z)$, where $\mu>0$ is an arbitrary positive constant. This 
function reaches minimum at $z^{*}=-0.303-0.648 i$ inside the white area, and this minimum is associated with the isotropic point where $F\left(z^{*}\right)=0$. Fig. 4 presents the map of the mean hydrostatic stress in the scaled form, i.e. the function $H(z)$ in expression $P(\mathrm{z})=\mu H(z)+v$, where $v>0$ is an arbitrary constant. The field of stress trajectories is shown in Fig. 5, from which the presence of the isotropic point is evident.

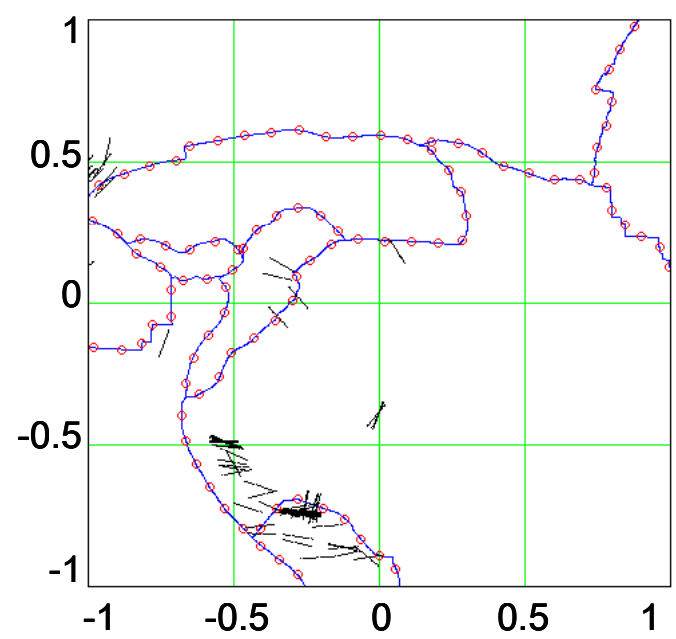

Figure 2: Computational domain and data; the boundaries of subdomains are shown by solid lines; circles show collocation points on the interfaces; short segments represent data on principal stress orientations.

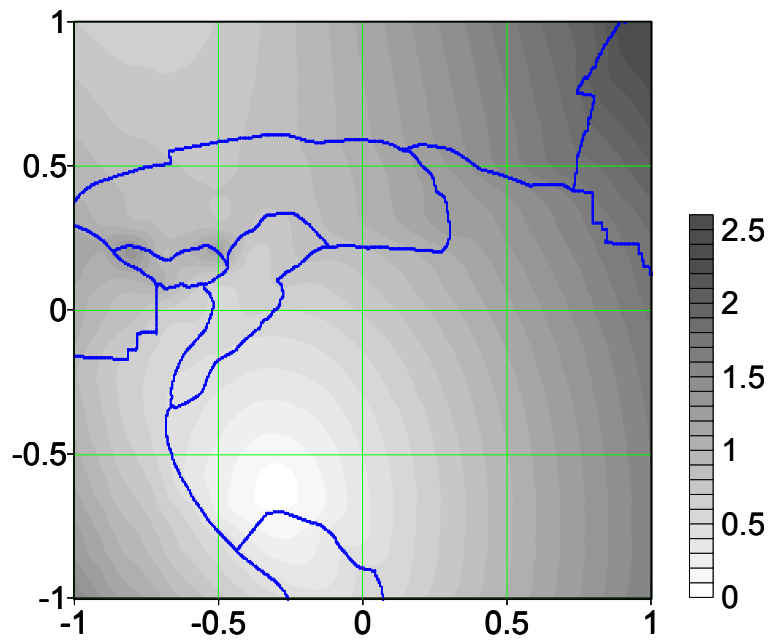

Figure 3: $\quad$ The map of maximum shear stress. 


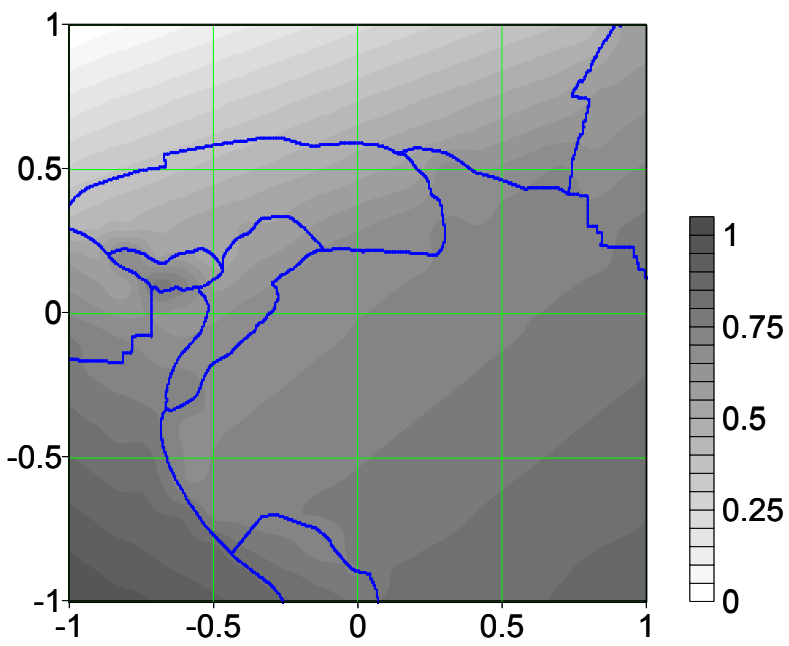

Figure 4: The map of mean hydrostatic stress.

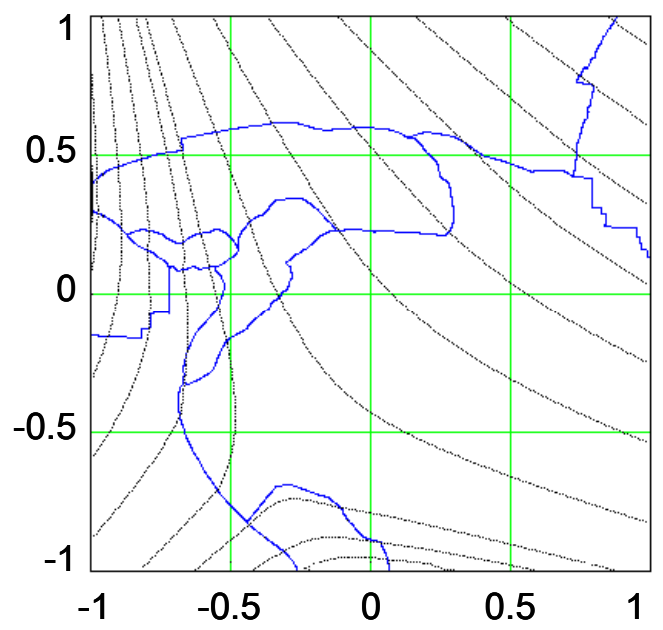

Figure 5: $\quad$ Stress trajectory field.

Although the fields of $\tau_{\max }(\mathrm{z})$ and $P(\mathrm{z})$ are discontinuous on the interfaces this is not clearly seen in Figs 3-4 because of interpolation used for plotting these maps. Stress trajectories also look smooth in the figure, however, the tangents to them presenting. the stress orientations suffer certain jump at the interfaces; at some collocation points the jump is up to $10^{\circ}$.

\section{Conclusion}

A numerical approach for the reconstruction of plane stress tensors in a composite domain with multiple interfaces is suggested in this paper. It provides 
essential tool for stress states identification by discrete data on principal directions and assumes neither boundary stresses nor displacements. The example considered in section 5 demonstrates that the proposed approach is capable for dealing with for geophysical applications with real data and complex geometries.

\section{Acknowledgement}

The author acknowledges the financial support of this study by EPSRC through the Research Grant EP/E032494/1.

\section{References}

[1] Galybin, A.N. and Sh.A. Mukhamediev, 2004. Determination of elastic stresses from discrete data on stress orientations, Int $J$ Solids and Structures, 41 (18-19), 5125-5142.

[2] Mukhamediev Sh.A., A.N. Galybin and B.H.G. Brady, 2006. Determination of stress fields in elastic lithosphere by methods based on stress orientations, Int J Rock Mech Min Sci, 43 (1), 66-88.

[3] Mukhamediev, Sh.A. and A..N. Galybin, 2006. Where and how did the ruptures of December 26,2004 and March 28, 2005 earthquakes near Sumatra originate? Doklady Earth Sciences. 406 (1), 52-55.

[4] Galybin, A. N., 2006. Stress fields in joined elastic regions: Modelling based on discrete stress orientations. In Situ Rock Stress - Lu, Li, Kjerholt \& Dahle (eds), Taylor \& Francis/Balkema, Leiden, 193-199.

[5] Heidbach, O., Tingay, M., Barth, A., Reinecker, J., Kurfeß, D. and B. Müller, 2008. The 2008 release of the World Stress Map (available online at www.world-stress-map.org)

[6] Bird, P., 2003. An updated digital model for plate boundaries. Geochem. Geophys. Geosyst., 4(3): 1027, doi:10.1029/2001GC000252.

[7] Zoback, M. L. and M. D. Zoback, 1989. Tectonic stress field of the conterminous United States, Mem. Geol. Soc. Am. 172: 523-539

[8] Mukhamediev Sh. A. and A. N. Galybin, 2007. Determination of Stresses from the Stress Trajectory Pattern in a Plane Elastic Domain; Mathematics and Mechanics of Solids; 12; 75.

[9] Muskhelishvili N.I., Some basic problems of the mathematical theory of elasticity, P. Noordhoff Ltd.: Groningen-Holland, 1953.

[10] Kuske A. and Robertson G., Photoelastic stress analysis, John Wiley \& Sons Ltd.: Bristol, 1974.

[11] Karakin, A.V., Mukhamediev, Sh. A., 1994. Singularities in nonuniform field of trajectories of the principal tectonic stresses. Izvestiya, Physics of the Solid Earth 29, 956-965. 